\section{TESTE DE CAMINHADA DE SEIS MINUTOS: DESEMPENHO DE CRIANÇAS COM EXCESSO DE PESO}

\author{
SIX-MINUTE WALKTEST:PERFORMANCE OF OVERWEIGHT CHILDREN
}

PRUEBA DE CAMINATA DE SEIS MINUTOS: RENDIMIENTO EN NIÑOS CON EXCESO DEPESO

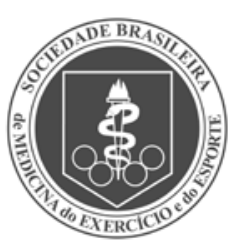

Artigo Original

Original Article Artículo Original

\author{
Fabíola Unbehaun Cibinello' \\ (Fisioterapeuta) \\ Carolina Cotrim Dal Pozzo' \\ (Fisioterapeuta) \\ Laís de Moura' \\ (Graduando em Fisioterapia) \\ Gissely Martins Santos' \\ (Graduando em Fisioterapia) \\ Dirce Shizuko Fujisawa' \\ (Fisioterapeuta)
}

1. Universidade Estadual de Londrina, Londrina, PR, Brasil.

\section{Correspondência:}

Alphaville Londrina 2 - Alameda Ipê Roxo, 357 - Conj. Res. Vivendas do Arvoredo, Lote Q40, Londrina, PR, Brasil. 86055-776.

fabi.cibinello@hotmail.com.

\section{RESUMO}

Introdução: O teste de caminhada de seis minutos (TC6') tem sido utilizado para avaliar a capacidade funcional do exercício em crianças. Objetivo: Comparar o desempenho no teste de caminhada de seis minutos em escolares na faixa etária entre 8 a 10 anos, conforme a classificação nutricional, com base em dois diferentes critérios. Métodos: A amostra de 226 crianças entre 8 a 10 anos foi dividida em três grupos (eutrófico, sobrepeso e obeso), de acordo com os critérios de classificação nutricional propostos pela Organização Mundial da Saúde (OMS) e pela International Obesity Task Force (IOTF) e avaliada por meio do teste de caminhada de seis minutos. Resultados: A prevalência de sobrepeso e obesidade na amostra foi de 42,48\% de acordo com o critério da OMS e 38,94\% pelo critério da IOTF. Não ocorreram diferenças estatisticamente significantes entre os grupos eutrófico, sobrepeso e obeso, quando os resultados do TC6 foram comparados, tanto na distância percorrida quanto no percentual do predito para a criança, de acordo com os valores de referência, e também de acordo com os dois critérios de classificação nutricional. A frequência cardíaca final do teste foi estatisticamente maior nos grupos sobrepeso e obeso, tanto pelo critério OMS ( $p=0,0015)$, quanto pelo critério IOTF $(p=0,0160)$. Conclusões: Na amostra estudada não houve diferença no desempenho do TC6 ${ }^{\prime}$ entre crianças eutróficas, com sobrepeso e obesas. Porém, a frequência cardíaca final do teste foi significantemente maior entre as crianças com excesso de peso. Os resultados ressaltam que medidas de prevenção, acompanhamento e tratamento são necessárias, promovendo hábitos saudáveis.

Descritores: obesidade; sobrepeso; atividade motora; saúde escolar; obesidade pediátrica.

\section{ABSTRACT}

Introduction: The six-minute walk test (6MWT) has been used to evaluate exercise functional capacity in children. Objective: To compare the performance in six-minute walk test in schoolchildren aged 8 to 10 years, according to nutritional classification based on two different criteria. Methods: The sample of 226 children aged 8 to 10 years was divided into three groups (eutrophic, overweight, and obese), according to the criteria of nutritional classification proposed by the World Health Organization (WHO) and by the International Obesity Task Force (IOTF) and assessed by the six-minute walk test. Results: The prevalence of overweight and obesity in the sample was $42.48 \%$ according to the WHO criteria and $38.94 \%$ according to the IOTF criteria. There were no statistically significant differences between the groups eutrophic, overweight, and obese, when the results of the 6MWT were compared, both in the distance covered and in the percentage predicted for children, according to the reference values, and according to the two criteria of nutritional classification. The final heart rate of the test was statistically higher in the overweight or obese groups, both by the WHO criteria $(p=0.0015)$ and by the IOTF criteria $(p=0.0160)$. Conclusions: I the studied sample, there was no difference in the performance of the 6MWT among eutrophic, overweight, and obese children. However, the final heart rate test was significantly higher among overweight children. The results emphasize that prevention, monitoring and treatment measures are necessary, promoting healthy habits.

Keywords: obesity; overweight; motor activity; school health; pediatric obesity.

\section{RESUMEN}

Introducción: La prueba de caminata de seis minutos (PC6'), ha sido utilizada para evaluar la capacidad funcional de ejercicio en niños. Objetivo: Comparar el rendimiento de la prueba de caminata de seis minutos en la población escolar entre 8 y 10 años de edad, de acuerdo con la clasificación nutricional con base en dos criterios diferentes. Métodos: La muestra de 226 niños de 8 a 10 años fue dividida en tres grupos (eutrófico, sobrepeso y obesidad) de acuerdo con los criterios de clasificación nutricional propuestos por la Organización Mundial de la Salud (OMS) y la International Obesity Task Force (IOTF), y evaluada por la prueba de caminata de seis minutos. Resultados: La prevalencia de sobrepeso y obesidad en la muestra fue de $42,48 \%$ de acuerdo con los criterios de la OMS y 38,94\% según la IOTF. No se encontraron diferencias significativas entre los grupos eutrófico, sobrepeso y obeso, cuando se compararon los resultados de la PC6'tanto en distancia y en porcentaje del predicho para niños, según los valores de referencia y también de acuerdo a los dos criterios de clasificación nutricional. La frecuencia cardiaca al final de la prueba, fue estadísticamente mayor en los grupos sobrepeso y obesidad, tanto en los criterios de la OMS ( $p=0,0015)$ y los de la IOTF $(p=0,0160)$. Conclusiones: En esta muestra no hubo ninguna diferencia en el rendimiento de la PC6 entre los niños eutróficos, con sobrepeso y obesidad. Sin embargo, la frecuencia cardiaca al final de la prueba fue 
significativamente mayor en los niños con exceso de peso. Los resultados señalan que la prevención, seguimiento y tratamiento son esenciales para promover hábitos saludables.

Descriptores: obesidad; sobrepeso; actividad motora; salud escolar; obesidad pediátrica.

\section{INTRODUÇÃO}

A Organização Mundial da Saúde (OMS) define obesidade como o acúmulo excessivo de gordura com potencial prejuízo a saúde. Trata-se de doença crônica que afeta tanto indivíduos adultos, quanto crianças e adolescentes. Em 1997, a OMS afirmou que os países em desenvolvimento estavam fadados a vivenciar as mesmas taxas de mortalidade por doenças crônicas e não transmissíveis que os países desenvolvidos. Tal fato estaria relacionado ao crescimento desenfreado da obesidade, que iria tornar-se cada vez mais prevalente na próxima década (WHO 1997)'. A prevenção e o tratamento da obesidade infantil é hoje o principal desafio global de saúde pública².

O sobrepeso e a obesidade são condições de etiologia multifatorial e sofrem influências de fatores biológicos, psicológicos, socioeconômicos e sócio-comportamentais, além de serem afetados pelo microambiente familiar e macroambiente de convívio da criança ${ }^{3}$. Nesse sentido, o aumento expressivo de crianças com sobrepeso e obesas está relacionado com as mudanças de hábitos da população, voltados para a dieta inadequada e a falta de atividade física.

Em estudo realizado com escolares, com o objetivo de determinar os fatores associados com a obesidade, revelou-se que a tendência ao sedentarismo é demonstrada pela associação entre o tempo de permanência sentado e o percentual de gordura corporal nas crianças ${ }^{4}$. Com a urbanização e o aumento da violência nas grandes cidades, o padrão de vida das crianças e adolescentes se modificou, aumentando o tempo despendido diante de televisores ou computadores, aumentando, assim, a inatividade física ${ }^{5}$.

Em crianças, o maior grau de atividade física contribui para meIhorar o perfil lipídico e a circunferência abdominal. As atividades de vida diária, como por exemplo, ir caminhando para a escola, podem auxiliar na diminuição de problemas metabólicos e doenças cardiovasculares ${ }^{6}$. O tempo gasto com atividade física de intensidade moderada a vigorosa está inversamente associado com a circunferência da cintura e a pressão arterial diastólica, e positivamente associada com o colesterol HDL?.

O teste de caminhada de seis minutos (TC6') tem sido usado, frequentemente, na última década para avaliar o esforço submáximo do indivíduo, que se mostra similar ao esforço para realizar as atividades de vida diária ${ }^{8}$. O TC6 é um método reprodutível, confiável e de baixo custo, que avalia a capacidade do esforço físico. Diferente de outros testes, o TC6' requer apenas um corredor de, aproximadamente, 30 metros de comprimento, e equipamentos simples para avaliar os parâmetros principais recomendados ${ }^{9}$. Recentemente, tem ocorrido grande interesse na obtenção de valores de referência para o TC6' na população pediátrica saudáve ${ }^{10-14}$. A identificação dos fatores que influenciam os resultados do teste possibilitará avaliar melhor o grau de comprometimento funcional em crianças com doenças crônicas ${ }^{15}$. Assim, é de grande importância que esse comprometimento funcional também seja avaliado em crianças com excesso de peso.

Existem poucos estudos na literatura que comparem os efeitos do sobrepeso e da obesidade com as crianças eutróficas em fase escolar. Além disso, não foram encontrados estudos que comparassem os resultados do TC6' entre crianças eutróficas, com sobrepeso e obesas. Portanto, o objetivo desse estudo foi comparar o desempenho no TC $6^{\prime}$ em crianças, na faixa etária entre oito a 10 anos, conforme a classificação nutricional com base nos critérios da IOTF e OMS.

\section{MÉTODOS}

O estudo transversal foi realizado em Escolas da Rede Municipal de Ensino de Londrina, PR, BR, tendo sido avaliadas crianças saudáveis na faixa etária entre oito a 10 anos. Os critérios de inclusão foram crianças de ambos os gêneros e que obtiveram autorização dos pais ou responsáveis para participação no estudo. Os critérios de exclusão foram crianças impossibilitadas de permanecerem na posição ortostática ou de deambular, e também aquelas que apresentassem alterações ortopédicas, doenças crônicas ou agudas, e as classificadas como baixo peso segundo os critérios da International Obesity Task Force (IOTF) ${ }^{16}$ ou $\mathrm{OMS}^{17}$. A amostra de conveniência foi constituída de 226 crianças. As escolas foram selecionadas por sorteio simples, tendo sido escolhidas cinco escolas, uma em cada região da cidade de Londrina-PR-BR. A Figura 1 traz as perdas e exclusões da amostra.

O projeto de pesquisa foi aprovado pelo Comitê de Ética em Pesquisa do Hospital Universitário/UEL protocolo n 2249961300005231, e a Secretaria Municipal de Educação foi contatada para estabelecimento de parceria. Os pais ou responsáveis pela criança foram esclarecidos quanto aos objetivos e a metodologia da pesquisa, incluindo os benefícios e incômodos e, posteriormente, foi solicitada a assinatura do termo de consentimento livre e esclarecido. As coletas foram realizadas no período de novembro de 2013 a junho de 2014. Após o término da coleta em cada escola, foi realizada devolutiva dos dados para a direção e professores. Também foram fornecidas orientações sobre possíveis encaminhamentos.

A massa corporal da amostra foi obtida por meio da balança da marca Marte, modelo LC 200, ano 2010, com capacidade Máxima de 200 kg e Mínima de 1000g. As crianças foram orientadas a permanecerem sem sapatos ou meias, vestindo somente o uniforme da escola, em ortostatismo, com os membros ao longo do corpo, posicionadas no centro da balança e olhando para frente. Já a estatura foi mensurada utilizando fita métrica de 150 centímetros (cm), com marcação de 0,1 $\mathrm{cm}$. As crianças foram orientadas a permanecerem eretas, joelhos em extensão, pés juntos, braços soltos ao longo do corpo e com os tornozelos, região glútea e ombros em contato com a parede. O IMC foi calculado pela fórmula peso (kg)/altura² (m).

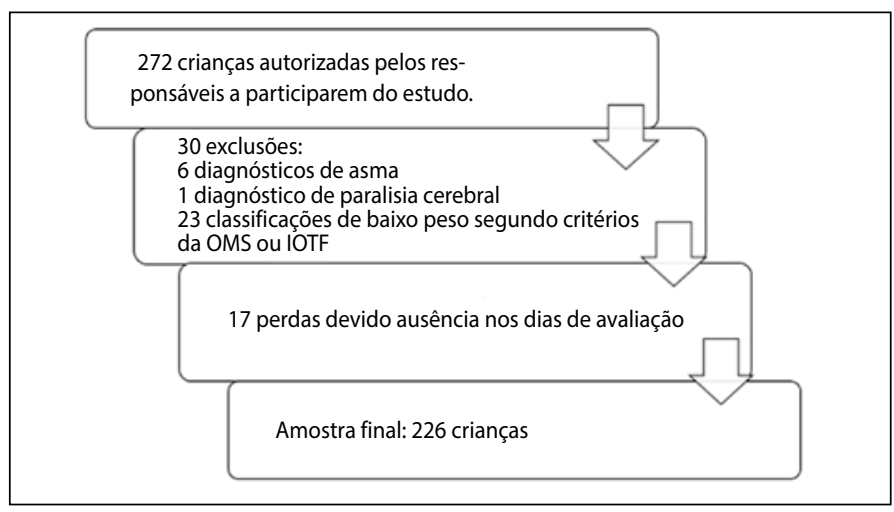

Figura 1. Fluxograma de exclusões e perdas da amostra. 
A classificação foi realizada pelos critérios da IOTF $^{16,18}$ e OMS ${ }^{17}$. O software Anthro Plus ${ }^{19}$ foi utilizado para calcular o escore $z$, a partir do peso, estatura e IMC. Os valores de score z menor que -2 significam baixo peso, entre -2 e +1 eutrofia, entre +1 e +2 sobrepeso e maiores que +2 obesidade. Foram utilizados os pontos de corte para a população infantil, a partir dos parâmetros no adulto (IMC $17 \mathrm{~kg} / \mathrm{m}^{2}$ - baixo peso, $18,5 \mathrm{~kg} / \mathrm{m}^{2}$ - eutrofia, $25 \mathrm{~kg} / \mathrm{m}^{2}$ - sobrepeso e $30 \mathrm{~kg} / \mathrm{m}^{2}$ - obesidade), considerando a idade e o gênero da criança, segundo os padrões propostos pela IOTF. A partir dessas classificações, as crianças foram divididas em três grupos: eutrófico, sobrepeso e obeso.

O TC6 foi realizado seguindo, rigorosamente, os critérios da American Thoracic Society. ${ }^{9}$ Antes de iniciar o TC6', todos os procedimentos e sua finalidade foram explicados aos participantes, com a finalidade de instruir e não ocorrer dúvida ou receio. O TC6' foi realizado em corredor plano de 30 metros de comprimento, com marcadores a cada três metros. No início e no final do corredor foram posicionados cones para sinalização. A saturação periférica de oxigênio e a frequência cardíaca foram monitoradas utilizando oxímetro portátil da marca Geratherm, modelo GT 300C203. A pressão arterial foi aferida com esfigmomanômetro da marca Nissei, modelo palm HT-1500. A sensação subjetiva de dispneia foi graduada de acordo com a escala de Borg modificada ${ }^{20}$. O monitoramento foi realizado antes, imediatamente após o término do teste (seis minutos) e durante o período de recuperação (cinco minutos após o término do teste) com os participantes na posição sentada. A distância percorrida durante o teste foi verificada por meio da contagem do número total de voltas efetuadas, e das marcações na pista de três em três metros. Durante a caminhada, as crianças foram incentivadas verbalmente e de forma padronizada pelos pesquisadores a cada um minuto. Cada criança realizou o teste duas vezes, com mínimo de 30 minutos de intervalo. Os dados analisados foram do segundo teste, em função do efeito aprendizado. Para determinar a distância predita para cada criança foi utilizada a formula proposta por Priesnitz et al. ${ }^{11}$, que considera a idade, massa corporal, a estatura e a diferença da frequência cardíaca antes e após o teste.

As variáveis foram testadas quanto à distribuição de normalidade por meio do teste de Shapiro-Wilk. Para verificar diferenças das variáveis entre os grupos eutrófico, sobrepeso e obeso e entre as idades (oito, nove e 10 anos) foi utilizado o teste de análise de variância (ANOVA) de medidas repetidas quando os dados foram normais, caso contrário, o teste Kruskal-Wallis. Para comparação dos dados entre meninos e meninas e entre os turnos (matutino e vespertino) foi utilizado o teste Mann Whitney, devido a não normalidade dos dados. A significância estatística foi estabelecida como $p<0,05$.

\section{RESULTADOS}

A amostra foi composta por 226 crianças, na faixa etária entre oito a 10 anos (Tabela 1). Dentre eles, 106 meninos (46,90\%) e 120 meninas (53,10\%), 111 (49,12\%) estudavam no turno matutino e 115 (50,88\%) no vespertino. Segundo os critérios da OMS, 76 (63,33\%) meninas foram classificadas como eutróficas, 23 (19,16\%) com sobrepeso e 21 (17,51\%) obesas; 54 (50,94\%) meninos foram classificados como eutróficos, 23 $(21,70 \%)$ com sobrepeso e 29 (27,36\%) obesos. A mediana do escore $z$ nas meninas foi $0,54(-0,15 ; 1,69)$ e nos meninos $0,99(-0,06 ; 2,08)$. O teste de Mann Whitney mostrou que não há diferença estatisticamente significante entre o escore $z$ entre os gêneros $(p=0,0761)$. Segundo os critérios da IOTF, 78 meninas (65,00\%) foram classificadas como eutróficas, 28 (23,33\%) com sobrepeso e 14 (11,67\%) obesas; 60 (56,60\%) meninos foram classificados como eutróficos, 30 (28,30\%) com sobrepeso e $16(15,10 \%)$ obesos. A mediana do IMC nas meninas foi de $17,45(15,69 ; 20,47)$ e nos meninos $18,01(16,06 ; 20,91)$. O teste de Mann Whitney mostrou que não há diferença estatisticamente significante entre o IMC e os gêneros ( $p=0,3023)$.

Não foram encontradas diferenças estatisticamente significantes, quando comparados os escores z entre os turnos matutino e vespertino ( $p=0,1615)$ e entre as idades oito, nove e 10 anos ( $p=0,3987)$. Também não ocorreram diferenças entre turnos quando comparado o IMC ( $p=$ 0,0840). Porém o teste de Kruskal-Wallis revelou que existe diferença estatística entre as idades em relação ao IMC $(p=0,0355)$. O teste de múltiplas comparações de Dunn revelou que a diferença se encontra nas idades de oito e 10 anos, com as respectivas medianas de 17,10 $(15,44 ; 20,29)$ e $19,00(16,32 ; 20,78)$.

Também não ocorreram diferenças estatisticamente significantes entre as diferentes regiões das escolas avaliadas de Londrina-PR e entre os grupos eutrófico, com sobrepeso e obeso, quando comparado os resultados do TC6,' tanto na distância percorrida quanto no percentual do predito para a criança, de acordo com os valores de referencia ${ }^{11}$, de acordo com ambos os critérios de classificação nutricional (Tabelas 2 e 3).

Na comparação da frequência cardíaca final no TC6'entre os três grupos, observou-se diferença estatisticamente significante, tanto pelo critério OMS ( $p=0,0015)$, quanto pelo critério IOTF $(p=0,0160)$ (Tabelas 4 e 5).

\section{DISCUSSÃO}

A prevalência de excesso de peso na amostra foi de $42,48 \%$ de acordo

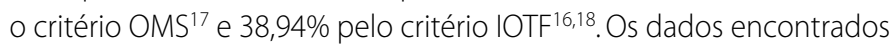
no estudo confirmam a tendência mundial para elevação acentuada da obesidade infantil. A OMS estima que 41 milhões de crianças no mundo tenham sido afetadas pelo sobrepeso e obesidade ${ }^{21}$.

A maior prevalência de excesso de peso no gênero masculino está de acordo com os dados da Pesquisa de Orçamentos Familiares 2008-2009-IBGE22. Não foram encontradas diferenças estatisticamente significantes quando comparado o escores z e IMC entre os turnos matutino e vespertino e entre as regiões da cidade de Londrina - PR. A não existência de diferença por região pode estar relacionadas às características semelhantes das escolas em termos de atividade, visto que todas são municipais, e às condições socioeconômicas das famílias.

Tabela 1. Caracterização da amostra.

\begin{tabular}{c|c|c}
\hline & Média \pm DP & $\begin{array}{c}\text { Mediana } \\
\text { (intervalo interquartílico) }\end{array}$ \\
\hline Idade (anos) & $8,74 \pm 0,70$ & $9,00(8,00 ; 9,00)$ \\
\hline Massa Corporal (Kg) & $35,58 \pm 9,29$ & $33,45(28,70 ; 40,93)$ \\
\hline Estatura (m) & $1,37 \pm 0,07$ & $1,37(1,32 ; 1,42)$ \\
\hline Escore z & $0,90 \pm 1,22$ & $0,75(-1,12 ; 1,95)$ \\
\hline IMC & $18,63 \pm 3,45$ & $17,62(15,88 ; 20,66)$ \\
\hline
\end{tabular}

Tabela 2. Desempenho no TC6'entre os grupos - critério da OMS.

\begin{tabular}{c|c|c}
\hline & $\begin{array}{c}\text { Distância percorrida } \\
\text { Média } \pm \text { DP }\end{array}$ & $\begin{array}{c}\text { \% alcançada do predito } \\
\text { Média } \pm \text { DP }\end{array}$ \\
\hline Eutrófico & $581,00 \pm 57,42$ & $98,76 \pm 9,03$ \\
\hline Sobrepeso & $588,9 \pm 68,01$ & $101,4 \pm 9,99$ \\
\hline Obeso & $573,10 \pm 52,48$ & $101,2 \pm 7,61$ \\
\hline & $p=0,4185$ & $p=0,1161$ \\
\hline
\end{tabular}

Tabela 3. Desempenho no TC6' entre os grupos - critério da IOTF.

\begin{tabular}{c|c|c}
\hline & $\begin{array}{c}\text { Distância percorrida } \\
\text { Média } \pm \text { DP }\end{array}$ & $\begin{array}{c}\text { \% alcançada do predito } \\
\text { Média } \pm \text { DP }\end{array}$ \\
\hline Eutrófico & $583,80 \pm 58,44$ & $99,11 \pm 9,15$ \\
\hline Sobrepeso & $586,60 \pm 62,26$ & $100,90 \pm 9,26$ \\
\hline Obeso & $561,40 \pm 50,11$ & $101,00 \pm 7,61$ \\
\hline & $p=0,1242$ & $p=0,3262$ \\
\hline
\end{tabular}


Tabela 4. Frequência Cardíaca final do TC6'entre os grupos - critério da OMS

\begin{tabular}{c|c}
\hline & $\begin{array}{c}\text { FC final do TC6 } \\
\text { Média } \pm \text { DP }\end{array}$ \\
\hline Eutrófico & $135,2 \pm 21,12$ \\
\hline Sobrepeso & $144,7 \pm 28,83^{\mathrm{a}}$ \\
\hline Obeso & $146,7 \pm 21,12^{\mathrm{a}}$ \\
\hline & $p=0,0015$ \\
\hline
\end{tabular}

avalores não diferem entre si de acordo com o teste de múltiplas comparações de Tukey.

Tabela 5. Frequência Cardíaca final do TC6'entre os grupos - critério da IOTF.

\begin{tabular}{c|c}
\hline & $\begin{array}{c}\text { FC final do TC6 } \\
\text { Média } \pm \text { DP }\end{array}$ \\
\hline Eutrófico & $136,6 \pm 21,53^{b}$ \\
\hline Sobrepeso & $142,6 \pm 22,09^{a b}$ \\
\hline Obeso & $148,3 \pm 23,37^{a}$ \\
\hline \multicolumn{2}{|c|}{$p=0,0160$} \\
\hline
\end{tabular}

A mediana do IMC foi significantemente maior na idade de 10 anos quando comparada com a idade de oito anos $(p=0,0355)$. Santos et al. ${ }^{23}$ concluíram que meninas na faixa etária de sete a 11 anos tendem a aumentar seu peso corporal com o passar da idade.

Estudos demonstraram que a habilidade de caminhar das crianças durante o teste é influenciada por variáveis, dentre elas, a frequência cardíaca. Esta apresenta um aumento significativo em resposta imediata ao TC6 ${ }^{\prime}$ e retorna aos valores basais após o primeiro minuto ${ }^{10-12,23,24}$. Na amostra estudada, foi observado que as crianças classificadas como sobrepeso e obesas apresentaram frequência cardíaca significantemente maior após o teste, quando comparadas com as eutróficas, em ambos os critérios de classificação nutricional. A literatura mostra que o escore z apresenta correlação inversa com a frequência cardíaca de recuperação após um teste de esforço em adolescentes saudáveis. ${ }^{25}$

Em recente pesquisa, foi evidenciado que os adolescentes com excesso de peso apresentam prejuízo na aptidão cardiorrespiratória não somente ao nível máximo, mas também no submáximo, quando comparados aos adolescentes com peso normal. ${ }^{26}$ Ao comparar crianças eutróficas sedentárias com obesas sedentárias, o estudo observou maior batimento cardíaco ao repouso e menor capacidade física por parte dos obesos. ${ }^{27}$ As crianças obesas apresentam modificações na variabilidade da frequência cardíaca, caracterizada por redução da atividade simpática e parassimpática. ${ }^{28}$

Na presente amostra, não existiram diferenças entre os grupos eutróficos, com sobrepeso e obeso quanto à distância percorrida e percentual do predito, em ambos os critérios de classificação nutricional.
Não foram encontrados estudos que comparassem os resultados do TC6' entre as diferentes classificações nutricionais. Petrelluzzi et al. ${ }^{27}$ analisaram o desempenho entre crianças obesas e não obesas utilizando o teste submáximo de esforço na esteira ergométrica, a principal diferença encontrada foi em relação ao tempo, o grupo obeso permaneceu realizando o teste até que fosse atingida a frequência cardíaca submáxima, que foi bem menor do que o grupo não obeso. Além disso, a distância percorrida revelou melhor capacidade física no grupo não obeso.

Em relação às considerações metodológicas, os dados foram coletados por cinco avaliadores, todos treinados conforme o padrão operacional dos procedimentos. O protocolo não incluiu padronização para atividades físicas e/ou refeições antes TC6', por se tratar de ambiente escolar, o controle desses aspectos não foram possíveis devido à programação acadêmica. Os dados em relação a sensação subjetiva de dispneia e fadiga, obtidos pela escala modificada de Borg ${ }^{20}$ poderiam auxiliar a compreensão da resposta da criança frente ao teste. Entretanto, estes dados não foram apresentados e analisados visto que as respostas das crianças não foram confiáveis. Recente revisão sistemática demonstrou que estudos têm comparado a escala de Borg ${ }^{20}$ com escalas adaptadas para crianças. Estes afirmam que as escalas adaptadas apresentam uma melhor performance, porém demonstram moderada correlação com os desfechos fisiológicos mensurados durante o exercício. ${ }^{29}$

\section{CONCLUSÃO}

Conclui-se que na amostra estudada não existem diferenças no desempenho do teste de caminhada entre eutroficos, com sobrepeso e obesos, tanto na distância percorrida quanto na porcentagem do predito para a criança, considerando os dois critérios de classificação nutricional. Todas as crianças alcançaram o predito para o teste, o que nos mostra que não foram encontradas limitações na capacidade funcional do esforço físico. Tal fato, provavelmente, está relacionado à faixa etária da amostra estudada ser precoce e que a obesidade ainda pode não ter influenciado o desempenho no teste. Entretanto, a frequência cardíaca final do teste foi, significantemente, maior entre as crianças com excesso de peso. O resultado mostra que, a resposta ao exercício acontece de maneira diferente nas crianças com sobrepeso e obesas. Em adição, pode-se citar que a frequencia de sobrepeso e obesidade encontrada na amostra corrobora com a atual literatura.

Todos os autores declararam não haver qualquer potencial conflito de interesses referente a este artigo.

CONTRIBUIÇÕES DE AUTORES: Cada autor contribuiu individual e significativamente para o desenvolvimento do manuscrito. FUC (0000-0002-8913-7331)* e CCDP (00000002-4172-4131)* realizaram a pesquisa bibliográfica e contribuíram com o conceito intelectual do estudo. FUC e DSF (0000-0001-8427-2860)* foram os principais contribuintes na redação do manuscrito e na análise estatística dos dados. FUC, CCDP, LM (0000-0002-8333-8319)*, GMS (0000-0002-9169-2254)*, e DSF coletaram e reuniram os dados do estudo e participaram ativamente da discussão dos resultados. DSF realizou a revisão e aprovação final do manuscrito. *ORCID (Open Researcher and Contributor ID).

\section{REFERÊNCIAS}

1. World Health Organization. Obesity: preventing and managing the global epidemic. Report of a WHO consultation. World Health Organ Tech Rep Ser. 2000;894:i-xii, 1-253.

2. World Health Organization. Population-based approaches to childhood obesity prevention. WHO Consulation on obesity; 2012.

3. Oliveira MA, Cerqueira EMM, Souza JS Oliveira AC. Sobrepeso e obesidade infantil: influência de fatores biológicos e ambientais em feira de Santana, BA. Arq Bras Endocrinol. Metab.2013;47(2):144-50.

4. Giugliano R, Carneiro EC. Factors associated with obesity in school children. J Pediatr (Rio J). 2004;80(1):17-22.

5. Rinaldi AEM, Pereira AF, Macedo CS, Mota JF, Burini RC. Contribuiçōes das práticas alimentares e inatividade física para o excesso de peso infantil. Rev Paul Pediatr. 2008;26(3):271-7.

6. Pizarro AN, Ribeiro JC, Marques EA, Mota J, Santos MP. Is walking to school associated with improved metabolic health? Int J Behav Nutr Phys Act. 2013;10:12.

7. Chaput JP, Saunders TJ, Mathieu MĖ, Henderson M, Tremblay MS, O'Loughlin J, et al. Combined associations between moderate to vigorous physical activity and sedentary behaviour with cardiometabolic risk factors in children. Appl Physiol Nutr Metab. 2013;38(5):477-83.

8. Solway S, Brooks D, Lacasse Y, Thomas S. A qualitative systematic overview of the measurement properties of functional walk tests used in the cardiorespiratory domain. Chest. 2001;119(1):256-70.

9. ATS Committee on Proficiency Standards for Clinical Pulmonary Function Laboratories.. ATS statement: guidelines for the six-minute walk test. Am J Respir Crit Care Med. 2002;166(1):111-7.

10. Geiger R, Strasak A, Treml B, Gasser K, Kleinsasser A, Fischer V, et al. Six-minute walk test in children and adolescents. J Pediatr. 2007;150(4):395-9.

11. Priesnitz CV, Rodrigues GH, Stumpf CS, Viapiana G, Cabral CP, Stein RT, et al. Reference values for the 6-min walk test in healthy children aged 6-12 years. Pediatr Pulmonol. 2009;44(12):1174-9.

12. Silva CM, Moraes WSL, Pin AS, Borges GF, Maciel TS. Valores de referência para teste de caminhada de 6 minutos em crianças saudáveis de 7 a 9 anos do Norte Brasileiro. Rev Gest Saúde. 2016;7(2):716-29.

13. Chen CA, Chang CH, Lin MT, Hua YC, Fang WQ, Wu MH, et al. Six-Minute Walking Test: Normal Reference Values for Taiwanese Children and Adolescents. Acta Cardiol Sin. 2015;31(3):193-201. 
14. Oliveira AC, Rodrigues CC, Rolim DS, Souza AA, Nascimento OA, Jardim JR, et al. Six-minute walk test in healthy children: is the leg length important? Pediatr Pulmonol. 2013;48(9):921-6.

15. Goemans N, Klingels K, van den Hauwe M, Boons S, Verstraete L, Peeters C, et al. Six-minute walk test: reference values and prediction equation in healthy boys aged 5 to 12 years. PLoS One. 2013;8(12):e84120.

16. Cole TJ, Flegal KM, Nicholls D, Jackson AA. Body mass index cut offs to define thinness in children and adolescents: international survey. BMJ. 2007;335(7612):194.

17. World Health Organization. Development of a WHO growth reference for school-aged children and adolescents. WHO Department of Nutrition; 2007.

18. Cole TJ, Bellizzi MC, Flegal KM, Dietz WH. Establishing a standard definition for child overweight and obesity worldwide: international survey. BMJ. 2000;320(7244):1240-3.

19. World Health Organization. Anthro Plus for personal computers Manual: Software for assessing growth of the world's. World Health Organization; 2009.

20. Borg GA. Psychophysical bases of perceived exertion. Med Sci Sports Exerc.1982;14(5):377-81.

21. World Health Organization. Report of the commission on ending childhood obesity. World Health Organization; 2016

22. Brasil. Ministério da Saúde. Instituto Brasileiro de Geografia e Estatística. Pesquisa de orçamentos familiares:
Antropometria e estado nutricional de crianças, adolescentes e adultos no Brasil. Rio de Janeiro: IBGE; 2010

23. Lammers AE, Hislop AA, Flynn Y, Haworth SG. The 6-minute walk test: normal values for children of 4-11 years of age. Arch Dis Child. 2008;93(6):464-8.

24. Santos AR, Zechin MJ, Paccagnella T, Parada K, Faria Junior M. Estudo comparativo da composição corporal entre idades de crianças pré-puberes do sexo feminino. Rev Bras Presc Fisiol Exerc. 2014;8(43):62-7.

25. Abu Hanifah R, Mohamed MN, Jaafar Z, Mohsein NA, Jalaludin MY, Majid HA, et al. The correlates of body composition with heart rate recovery after step test: an exploratory study of Malaysian adolescents. PLoS One. 2013;8(12):e82893.

26. Gomes CB, Carletti L,Perez AJ. Desempenho em teste cardiopulmonar de adolescentes: peso normal e excesso de peso. Rev Bras Med Esporte. 2014; 20(3):195-9.

27. Petrelluzzi KFS, Kawamura M, Paschoal, MA. Avaliação funcional cardiovascular de crianças sedentárias obesas e não obesas. Rev Cienc Med Campinas. 2004;13:127-36.

28. Vanderlei LC, Pastre CM, Freitas Júnior IF, Godoy MF. Analysis of cardiac autonomic modulation in obese and eutrophic children. Clinics (Sao Paulo). 2010;65(8):789-92.

29. Rodríguez I, Zambrano L, Manterola C. Criterion-related validity of perceived exertion scales in healthy children: a systematic review and meta-analysis. Arch Argent Pediatr. 2016;114(2):120-8. 